\title{
SDSS J094635.06+101706.1: a redshift one, very radio-loud, $\gamma$-ray emitting narrow-line Seyfert 1 galaxy
}

\author{
Su Yao ${ }^{1,2 \star}$, S. Komossa ${ }^{3}$, Wen-Juan Liu ${ }^{4,5}$, Weimin Yi ${ }^{4,5,6}$, Weimin Yuan ${ }^{7}$, \\ Hongyan $\mathrm{Zhou}^{8,9}$, Xue-Bing $\mathrm{Wu}^{1,10}$ \\ ${ }^{1}$ Kavli Institute for Astronomy and Astrophysics, Peking University, Beijing 100871, China \\ ${ }^{2}$ National Astronomical Observatories, Chinese Academy of Sciences, Beijing 100012, China \\ ${ }^{3}$ Max-Planck Institut für Radioastronomie, Auf dem Hügel 69, 53121 Bonn, Germany \\ ${ }^{4}$ Yunnan Observatories, Chinese Academy of Sciences, Kunming, Yunnan 650011, China \\ ${ }^{5}$ Key Laboratory for the Structure and Evolution of Celestial Objects, Chinese Academy of Sciences, Kunming 650011, China \\ ${ }^{6}$ Department of Astronomy \& Astrophysics, The Pennsylvania State University, 525 Davey Lab, University Park, PA 16802, USA \\ ${ }^{7}$ Key Laboratory of Space Astronomy and Technology, National Astronomical Observatories, Chinese Academy of Sciences, Beijing 100012, China \\ ${ }^{8}$ CAS Key Laboratory for Research in Galaxies and Cosmology, Department of Astronomy, USTC, Hefei, Anhui 230026, China \\ ${ }^{9}$ SOA Key Laboratory for Polar Science, Polar Research Institute of China, 451 Jinqiao Road, Shanghai 200136, China \\ ${ }^{10}$ Department of Astronomy, School of Physics, Peking University, Beijing 100871, China
}

Accepted XXX. Received YYY; in original form ZZZ

\begin{abstract}
As hybrids of narrow-line Seyfert 1 (NLS1) galaxies and blazars, $\gamma$-ray emitting NLS1s are important probes of jet physics in the high Eddington-ratio regime. Only very few of them are known to date; the majority of them below redshift $z=0.5$. Here we present the identification of the $\gamma$-ray emitting AGN TXS 0943+105 (SDSS J094635.06+101706.1) as a high-redshift NLS1 galaxy. It turns out to be one of the radio-loudest NLS1s known, highly variable at all wavelengths, and shows widely extended radio emission at a (projected) $>100 \mathrm{kpc}$ scale. It is a known strong $\gamma$-ray emitter with a luminous flare reported previously. At redshift $z=1.004$, this is the most distant $\gamma$-NLS1 known to date.
\end{abstract}

Key words: galaxies: active - galaxies: nuclei - galaxies: Seyfert - accretion, accretion discs - galaxies: jets

\section{INTRODUCTION}

Radio-loud and $\gamma$-ray emitting narrow-line Seyfert 1 (NLS1) galaxies are important new probes of the formation and evolution of radio jets, in a regime different from classical blazars (see a review by Komossa 2018). NLS1s are a subgroup of AGN with extreme properties in the optical and X-ray band, and therefore stand out in AGN correlation space (e.g. Boroson \& Green 1992; Grupe et al. 2010; $\mathrm{Xu}$ et al. 2012). They harbor supermassive black holes (SMBHs) of relatively low masses, accreting at a high rate, and therefore represent a class of AGN which are rapidly growing their SMBHs in the local universe.

While only studied in the optical and X-ray regime for some decades and preferentially radio-quiet, nevertheless a population of radio-loud, jet-emitting NLS1 galaxies was identified (Komossa et al. 2006; Yuan et al. 2008). These combine the properties of blazars on the one hand, and NLS1 galaxies on the other hand. With Fermi/LAT, it was discovered that a few of the jetted NLS1 galaxies emit luminous $\gamma$-ray radiation, highly variable in some cases, and

^ E-mail: yaosu@pku.edu.cn independently confirming the presence of powerful jets in some NLS1 galaxies (Abdo et al. 2009b). Meanwhile, about $15 \gamma$-ray emitting NLS1s have been identified (Abdo et al. 2009a; Abdo et al. 2009a; Foschini 2011; D'Ammando et al. 2012; D'Ammando et al. 2015; Yao et al. 2015; Yang et al. 2018; Paliya et al. 2018; Lähteenmäki et al. 2018; see Komossa 2018 and Romano et al. 2018 for reviews).

NLS1 galaxies as a group are spectroscopically defined by the small widths of their broad $\mathrm{H} \beta$ emission lines, with full width at half maximum (FWHM) typically taken to be less than $2000 \mathrm{~km} \mathrm{~s}^{-1}$, strong Fe II emission complexes and weak forbidden lines (Osterbrock \& Pogge 1985). Most NLS1s have been reported at $z \lesssim 0.8$, including the ones detected at $\gamma$-rays, as their identification requires $\mathrm{H} \beta$ falling into the spectral coverage of large surveys. An exception is SDSS J1222+0413 at $z=0.966$ (Yao et al. 2015).

Here, we report the identification of TXS $0943+105$ (SDSS J094635.06+101706; J0946+1017 hereafter) at redshift $z=1.004$ as a new very radio-loud NLS1 galaxy which is also $\gamma$-ray detected (Acero et al. 2015). The optical identification is based on the Sloan Digital Sky Survey-III Baryon Oscillation Spectroscopic Survey (SDSS-BOSS, Dawson et al. 2013), which for the first time in- 
cluded the $\mathrm{H} \beta$ regime (Section 2) because of the broader wavelength range covered. Multi-wavelength follow-up observations are presented in Section 3, while Section 4 provides the discussion and conclusions. Throughout this paper we use a $\Lambda$ CDM cosmology with $H_{0}=70 \mathrm{~km} \mathrm{~s}^{-1} \mathrm{Mpc}^{-1}, \Omega_{\Lambda}=0.73$ and $\Omega_{\mathrm{M}}=0.27$.

\section{SPECTROSCOPY}

\subsection{Observations}

The optical spectrum of J0946+1017 was obtained on January 27, 2012 (MJD 55953) during SDSS-III's Baryon Oscillation Spectroscopic Survey (BOSS, Dawson et al. 2013) by the Sloan $2.5 \mathrm{~m}$ telescope equipped with a spectrograph which covers a wavelength range of 3600-10400 $\AA$ and has a spectral resolution of $R=1560$ 2650. We retrieved the calibrated 1D spectrum from the SDSS archive.

During the 2016A cycle of the Telescope Access Program of China, we obtained a near-infrared (NIR) spectrum of J0946+1017 using the TripleSpec spectrograph mounted on the Palomar $5 \mathrm{~m}$ Hale telescope on April 20 (UT), 2016 (MJD 57498). The spectrograph provides a wavelength coverage from 0.9 to $2.46 \mu \mathrm{m}$ at a resolution of 1.4-2.9 $\AA$ with two gaps at approximately 1.35 and $1.85 \mu \mathrm{m}$ owing to telluric absorption bands (Wilson et al. 2004). For optimal removal of the strong sky background in the NIR, we observed target (A) and off-target (B) positions in an A-B-B-A dither pattern along the slit with four exposures of $300 \mathrm{~s}$. The sky was clear with seeing $\sim 1.2^{\prime \prime}$ and the slit-width was fixed to $1^{\prime \prime}$. The data reduction, including flux calibration and telluric correction, was carried out with the Interactive Data Language (IDL)-based package SpexTool (Cushing et al. 2004), as described in detail in Zuo et al. (2015).

\subsection{Data Analysis}

Before the spectral fitting, both BOSS and TripleSpec spectra are corrected for Galactic extinction with $E(B-V)=0.025 \mathrm{mag}$ (Schlafly \& Finkbeiner 2011) and an $R_{V}=3.1$ extinction law, and then are transformed into the source rest frame at a redshift $z=1.004$ provided by the SDSS spectroscopic pipeline. The fitting is based on IDL routines in the MPF IT package (Markwardt 2009), which performs a $\chi^{2}$ minimization using the LevenbergMarquardt method. Due to flux variability of the source and nonsimultaneity of the spectroscopy, the SDSS-BOSS and TripleSec spectra had to be re-scaled. We fit the BOSS spectrum using a single power law in the fitting windows which are thought to be free of any strong emission lines: [3020, 3030] $\AA,[3790,3810] \AA$, [4200, 4210] $\AA$ and [5080, 5100] $\AA$ (Vanden Berk et al. 2001). Then the TripleSpec spectrum is scaled so that the spectrum in the range [5300, 6400] $\AA$ matches the best-fit single power law. The BOSS spectrum and the scaled TripleSpec spectrum are combined into one spectrum (Figure 1). We use this combined spectrum in the following analysis.

In order to measure the primary emission lines, we adopt a similar procedure as in Yao et al. (2015), which models the emission lines and their underlying continuum simultaneously. We perform spectral fitting separately for the Balmer lines in the optical and the $\mathrm{Mg}$ II line in the ultraviolet. During the fitting procedure, a pseudo-continuum consisting of a simple power law and Fe II multiplets is used. The optical and ultraviolet Fe II are modelled using templates provided in Véron-Cetty et al. (2004) and Tsuzuki et al. (2006), respectively.

The broad Balmer lines including $\mathrm{H} \alpha$ were modelled with either a Lorentzian or a concentric double-Gaussian profile. Narrow lines potentially present in the spectrum, including possible narrow Balmer lines, [OIII] $\lambda \lambda 4959,5007$ and [NII] $\lambda \lambda 6548,6583$ are described by a single Gaussian profile with FWHM $\leqslant 900 \mathrm{~km} \mathrm{~s}^{-1}$. The broad Balmer lines are constrained to have the same width and redshift, and a similar constraint is set for all the narrow lines. Then the Balmer emission lines are fitted simultaneously with a pseudocontinuum in the range of $[4160,5100] \AA$ and $[6000,6800] \AA$.

To measure the $\mathrm{Mg}$ II line, line profile and pseudo-continuum are fitted simultaneously to the spectrum in the range of [2200, 3100] $\AA$. We fit the $\operatorname{Mg} \operatorname{II} \lambda \lambda 2796,2803$ doublet using a similar model as in Wang et al. (2009). Each line of the doublet is modeled with one broad and one narrow component. The broad component is a truncated five-parameter Gauss-Hermite profile (e.g. van der Marel \& Franx 1993). Both broad components are assumed to have the same width and redshift, and their flux ratio is constrained between 2:1 and 1:1 (Laor et al. 1997). The narrow component is a single Gaussian profile with constraint of FWHM $\leqslant 900 \mathrm{~km} \mathrm{~s}^{-1}$. The redshift and line flux ratio of the two narrow components are constrained following the same prescription as for the broad components. We find, however, that addition of narrow components in $\mathrm{Mg}$ II is not needed.

No other narrow lines are detected in the spectrum, either. The Balmer lines can be similarly well fitted with either a Lorentzian or a double Gaussian profile, with $\operatorname{FWHM}\left(\mathrm{H} \beta_{\text {broad }}\right)=1974 \pm$ $51 \mathrm{~km} \mathrm{~s}^{-1}$ for a Lorentzian and $2199 \pm 30 \mathrm{~km} \mathrm{~s}^{-1}$ for a doubleGaussian profile, respectively. These measurements imply a NLS1 classification of J0946+1017. This classification is independently confirmed by the faintness of [O III] as well as the very strong Fe II emission complexes, with $R_{4570} \equiv \mathrm{Fe}$ II $\lambda 4570 / \mathrm{H} \beta_{\text {total }} \approx 1.0$, where Fe II $\lambda 4570$ is the flux of Fe II integrated from $4434 \AA$ to $4684 \AA$ (e.g. Véron-Cetty et al. 2001). The fitting results are summarized in Table 1.

\section{MULTI-WAVELENGTH PROPERTIES}

\subsection{Radio emission}

J0946+1017 was detected by several radio surveys. Its highest precision radio coordinate from Very Long Baseline Radio Interferometry observations (VLBI, RA=09h46m35.1s, Del=+10 $17^{\prime} 06^{\prime \prime} .1$, Fey et al. 2015) agrees within better than 0.1 arcsec with its Gaia (RA=09h46m35.1s, Del $=+10^{\circ} 17^{\prime} 06^{\prime \prime} .1$, Andrae et al. 2018) and SDSS coordinates. The Texas Survey measured a flux density of $505 \pm 28 \mathrm{mJy}$ at $365 \mathrm{MHz}$ with a spectral index of $\alpha_{\text {rad }} \sim-0.5$ $\left(S_{v} \propto v^{\alpha}\right)$ estimated between $335-380 \mathrm{MHz}$, but the error in this spectral index is naturally high given the small frequency range involved (Douglas et al. 1996). The non-simultaneous observations by the Green Bank telescope imply flux densities of $379 \mathrm{mJy}$ at $1.4 \mathrm{GHz}$ and $294 \mathrm{mJy}$ at $4.85 \mathrm{GHz}$, respectively, and the spectral index between 1.4 and $4.85 \mathrm{GHz}$ is flat with $\alpha_{\text {rad }} \sim-0.2$ (Becker et al. 1991; White \& Becker 1992). In the higher frequency range, J0946+1017 was observed at $8.4 \mathrm{GHz}$ with the Very Large Array (VLA) within the framework of the Combined Radio All-Sky Targeted Eight GHz Survey (CRATES, Healey et al. 2007) with $\sim 293$ mJy flux density and at $22 \mathrm{GHz}$ with the VLBI Exploration of Radio Astrometry (VERA) instrument with 90 mJy flux density (Petrov et al. 2007). 


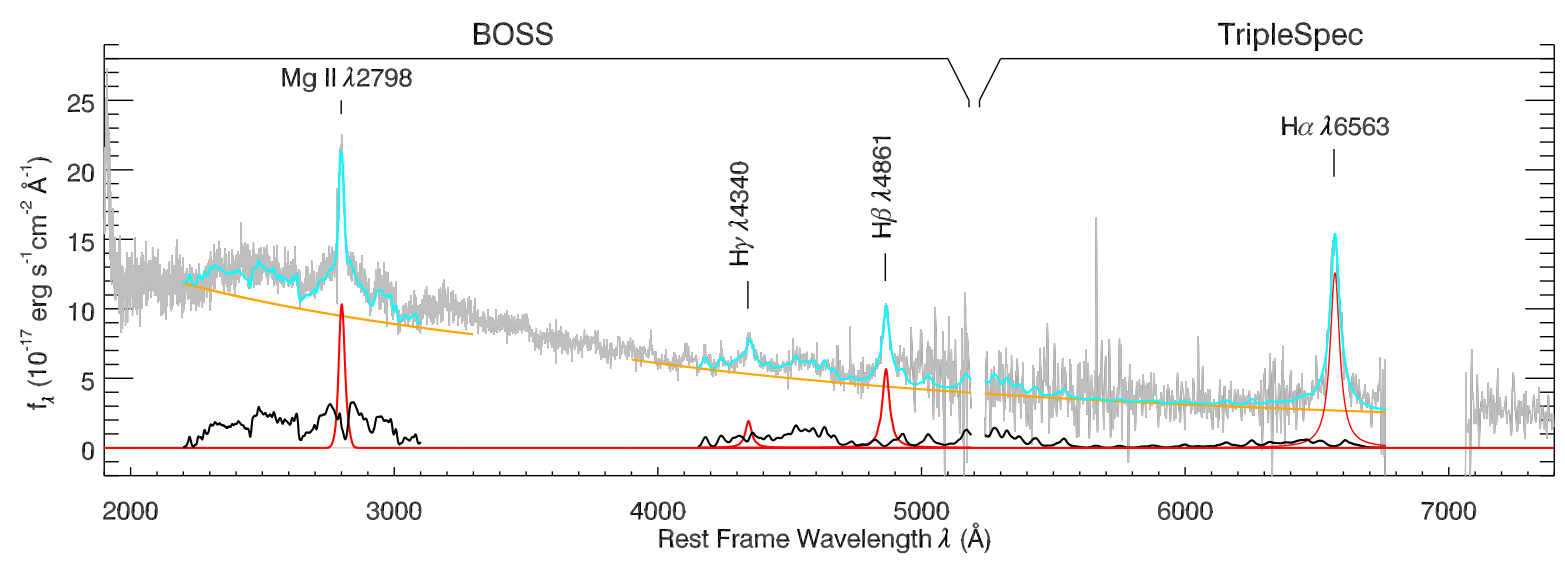

Figure 1. The combined rest-frame spectrum (grey) of J0946+1017 after correction for Galactic extinction. The bottom black curves represent the best-fit Fe II multiplets underneath the Mg II and Balmer lines. The orange curves represent the best-fit single power law model and the red curves provide the best-fit broad emission line models. The cyan curves represent the combination of the models.

Table 1. Fit results for the main emission lines.

\begin{tabular}{|c|c|c|c|c|c|c|c|}
\hline & $\begin{array}{c}\text { FWHM }\left(\mathrm{H} \beta_{\text {broad }}\right) \\
{\left[\mathrm{km} \mathrm{s}^{-1}\right]}\end{array}$ & $\begin{array}{c}f\left(\mathrm{H} \alpha_{\text {broad }}\right) \\
{\left[\mathrm{erg} \mathrm{s}^{-1} \mathrm{~cm}^{-2}\right]}\end{array}$ & $\begin{array}{c}f\left(\mathrm{H} \beta_{\text {broad }}\right) \\
{\left[\mathrm{erg} \mathrm{s}^{-1} \mathrm{~cm}^{-2}\right]}\end{array}$ & $\begin{array}{c}f\left(\mathrm{H} \gamma_{\text {broad }}\right) \\
{\left[\mathrm{erg} \mathrm{s}^{-1} \mathrm{~cm}^{-2}\right]}\end{array}$ & $R_{4570}$ & $\begin{array}{c}\left.\text { FWHM(Mg II } \text { broad }_{\text {of }}\right) \\
{\left[\mathrm{km} \mathrm{s}^{-1}\right]}\end{array}$ & $\begin{array}{l}f\left(\mathrm{Mg}_{\text {I }} \text { broad }\right. \\
{\left[\mathrm{erg} \mathrm{s}^{-1} \mathrm{~cm}^{-2}\right]}\end{array}$ \\
\hline Lorent & $1974 \pm 51$ & $(17.24 \pm 0.42) \times 10^{-15}$ & $(5.76 \pm 0.20) \times 10^{-15}$ & $(1.75 \pm 0.11) \times 10^{-15}$ & $0.90 \pm 0.08$ & \multirow{2}{*}{$2919 \pm 196$} & \multirow{2}{*}{$(6.52 \pm 0.21) \times 10^{-15}$} \\
\hline Double-Gaussian & $2199 \pm 30$ & $(17.58 \pm 1.02) \times 10^{-15}$ & $(5.44 \pm 0.41) \times 10^{-15}$ & $(2.32 \pm 0.06) \times 10^{-15}$ & $1.04 \pm 0.04$ & & \\
\hline
\end{tabular}

Notes. FWHMs are not corrected for instrumental resolution.

The Faint Images of the Radio Sky at Twenty-Centimeters (FIRST, Becker et al. 1995) survey image of J0946+1017, with rms of $0.135 \mathrm{mJy}$, reveals a two-component morphology as shown in Figure 2. The core component is consistent in position with J0946+1017 in the optical and has an integrated flux density of $301.9 \mathrm{mJy}$, while the secondary component is $\sim 15^{\prime \prime}$ (i.e., projected distance of $120 \mathrm{kpc}$ at rest frame) separated in north-west direction from the core and has an integrated flux density of $12.8 \mathrm{mJy}$ and a side-lobe probability of only $P(S)=0.025$ (Helfand et al. 2015).

In addition, Very Long Baseline Array (VLBA) observations of J0946+1017 at $5 \mathrm{GHz}$ revealed a core brightness temperature of $2.4 \times 10^{10} \mathrm{~K}$ and a discernible jet feature with an angular extent less than 6 milliarcseconds, implying a jet on tens of parsec scale (Linford et al. 2012). By using the Green Bank flux density $S_{4.85 \mathrm{GHz}}=$ 294 mJy (White \& Becker 1992) and the SDSS $g$-magnitude, we calculate the radio loudness of J0946+1017 as $\log R \approx 3.7$ after the $k$-correction with radio spectral index $\alpha_{\text {rad }} \sim-0.2$ (White \& Becker 1992) and optical spectral index $\alpha_{\text {opt }} \approx-0.35$ obtained from the spectral fitting (Section 2.2). Even considering radio variability, J0946+1017 is still an extremely radio-loud AGN, and one of the radio-loudest among known NLS1 galaxies.

\subsection{Near-infrared and optical variability}

J0946+1017 was observed with the Wide-field Infrared Survey Explorer (WISE; Wright et al. 2010) in four bands $w 1, w 2, w 3$ and $w 4$ centred at 3.4, 4.6, 12 and $24 \mu \mathrm{m}$, respectively. We employed the ALLWISE and NEOWISE database (Mainzer et al. 2014). Due to the depletion of the satellite's cooling material (Mainzer et al. 2014), we only constructed the Near-infrared (NIR) light curves of J0946+1017 in $w 1$ and $w 2$ which correspond to J0946+1017 emission at $\sim 1.7 \mu \mathrm{m}$ and $2.3 \mu \mathrm{m}$ at rest frame, respectively, We use $w 1$ and $w 2$ photometry data with $\mathrm{S} / \mathrm{N}>10$ and reduced $\chi^{2}$ lower than 2

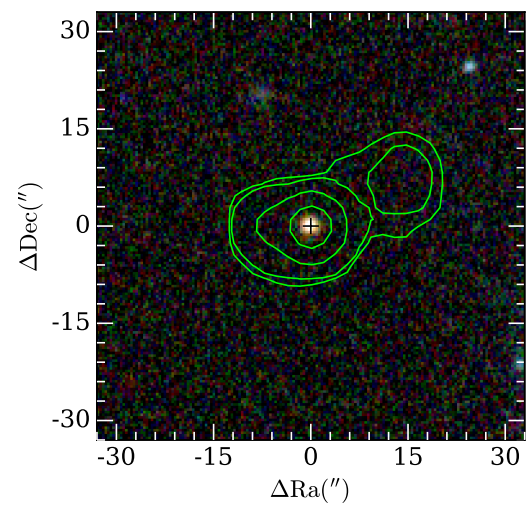

Figure 2. Pseudo-color RGB image created from SDSS $i-r$ - $g$ band images centered at J0946+1017's position (black '+'), overlaid with the FIRST $1.4 \mathrm{GHz}$ image (green contours). The contours are plotted as $(1,2,22,125) \times 6 \sigma$.

based on profile fitting ${ }^{1}$ in which case $\sim 7 \%$ of the data points are discarded.

The $w 1$ and $w 2$ light curves of $\mathrm{J} 0946+1017$ are displayed in Figure 3. Intra-day variability of $w 1$ flux is detected with $p$-values of $P<0.5$ percent using the $\chi^{2}$-test against the null hypothesis of no variation in 2015 November. The rapid variability restricts the size of the emitting region to be much smaller than the scale of the torus, implying a jet origin (see also Jiang et al. 2012; Gabanyi et al. 2018a; Rakshit et al. 2019). On timescale of years, the NIR

\footnotetext{
1 The image processing and data quality details can be found in http://wise2.ipac.caltech.edu/docs/release/ allsky/expsup/.
} 


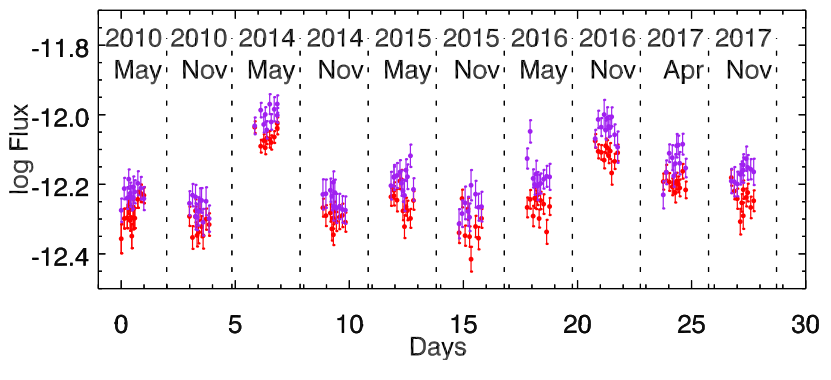

Figure 3. WISE light curves in the $w 1$ (red) and $w 2$ (purple) band in units of ergs s${ }^{-1} \mathrm{~cm}^{-2}$ converted from profile-fit magnitudes (Wright et al. 2010). The MJDs of the light curves are offset for visual clarity.

flux varied by nearly 0.4 dex from May 2014 to November 2015 . We also explore the color variation $(w 1-w 2)$. But no significant color variability has been found.

We have checked the archival photometry of the Catalina Real Time Transient Survey (CRTS, Drake et al. 2009) and the Panoramic Survey Telescope \& Rapid Response System (PanSTARRS, Chambers et al. 2016) of J0946+1017 in the optical bands. CRTS has monitored J0946+1017 from May 2005 to January 2014. In spite of large uncertainties, the CRTS light curve reveals clear variability of $\mathrm{J} 0946+1017$ in the synthetic $V$-band, which brightened by $\sim 0.5$ magnitude from 2006 to 2010 and then became fainter again. Pan-STARRS monitored J0946+1017 in the grizy-bands between January 2010 and April 2014, and it also captured the slow decrease of the source's brightness after 2010 in all five bands.

\subsection{High-energy emission}

\subsubsection{X-ray emission}

The ROSAT All-Sky Survey (RASS; Voges et al. 1999) performed in 1990-1991 has covered the position of J0946+1017. Following the method in Yuan et al. (2014), we obtain a flux upper limit of $1.7 \times$ $10^{-13} \mathrm{erg} \mathrm{s}^{-1} \mathrm{~cm}^{-2}$ in the band $0.1-2.4 \mathrm{keV}$ at the $99 \%$ confidence level.

We proposed an XMM-Newton pointing observation of $60 \mathrm{ks}$ on J0946+1017 which was carried out on 2017 November 3 (ObsID 0800040101). The data are reduced with the XMM-Newton SCIENCE ANALYSIS SYSTEM ${ }^{2}$ (VERSION 15) following standard procedures. The source position in the X-rays (R.A. $=09: 46: 35.08$, Dec $=+10: 17: 06.04)$ is well consistent with that in SDSS image within 1 arcsec. The source events are extracted from a circle of $40^{\prime \prime}$ radius centred at source position for data from the PN CCD arrays (Strüder et al. 2001), while the background events are extracted from a circle of the same radius in a source-free region nearby. The spectrum is binned to contain at least 25 counts per bin required for $\chi^{2}$ analysis. XSPEC V.12.9.0 (Arnaud 1996) is used for spectral modeling over the $0.3-10 \mathrm{keV}$ energy range. We find that the spectrum can be well fitted $\left(\chi^{2} /\right.$ dof $\left.=193 / 183\right)$ by a single power law and Galactic hydrogen absorption with column density of $N_{\mathrm{H}}^{\mathrm{Gal}}=2.45 \times 10^{20} \mathrm{~cm}^{-2}$ (Kalberla et al. 2005). The fit is slightly improved $\left(\chi^{2} /\right.$ dof $\left.=172 / 181\right)$ if we add a black body component, and we find a temperature of $k T_{\mathrm{bb}}=0.23_{-0.08}^{+0.07} \mathrm{keV}$ at

\footnotetext{
2 https://www.cosmos.esa.int/web/xmm-newton/ what-is-sas
}

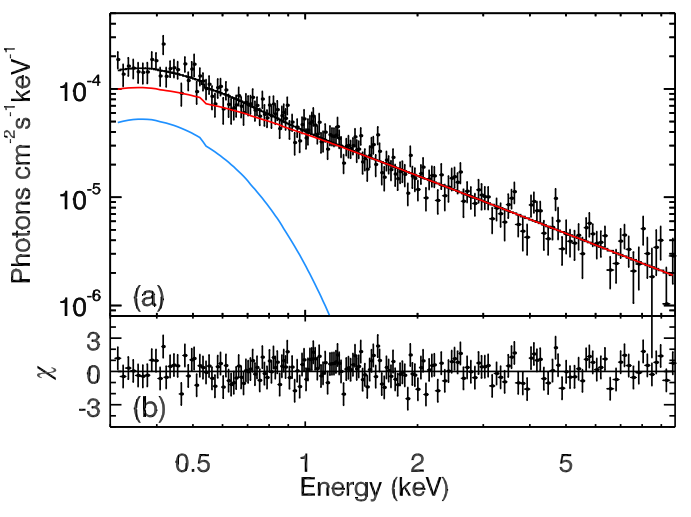

Figure 4. (a) The $X M M-N e w t o n / \mathrm{PN}$ unfolded spectrum and the best-fit model (black curve) consisting of power law (red) and black body (blue). (b) Residuals of the power law plus black body model fitted to the spectrum.

rest frame (Figure 4). The best-fit photon index is $\Gamma=1.35_{-0.09}^{+0.08}$, which is relatively flat among the population of normal radio-quiet NLS1s (e.g. Grupe et al. 2010), but similar to other $\gamma$-ray emitting NLS1s $(\Gamma<2$, e.g. Abdo et al. 2009b). The total unabsorbed flux based on this model is $f_{0.3-10 \mathrm{keV}}=4.04 \times 10^{-13} \mathrm{erg} \mathrm{s}^{-1} \mathrm{~cm}^{-2}$ and $f_{0.1-2.4 \mathrm{keV}}=1.38 \times 10^{-13} \mathrm{erg} \mathrm{s}^{-1} \mathrm{~cm}^{-2}$, with corresponding luminosities of $2.2 \times 10^{45} \mathrm{erg} \mathrm{s}^{-1}$ and $7.6 \times 10^{44} \mathrm{erg} \mathrm{s}^{-1}$, respectively.

\subsection{2 $\gamma$-ray emission}

$\gamma$-ray emission from the direction of J0946+1017 was reported by Fermi/LAT. The source 4FGL J0946.6+1016 was identified with the radio source TXS $0943+105$ with association probability $>99.9 \%$ (The Fermi-LAT collaboration 2019). The significance of the $\gamma$-ray detection increases from $7 \sigma$ in the LAT 1year point source catalog (1FGL, Abdo et al. 2010) to $46 \sigma$ in the LAT 8-year point source catalog (4FGL, The Fermi-LAT collaboration 2019). The flux (luminosity) between $100 \mathrm{MeV}$ and $100 \mathrm{GeV}$ also increases from $8.2 \times 10^{-12} \mathrm{erg} \mathrm{s}^{-1} \mathrm{~cm}^{-2}\left(4.5 \times 10^{46} \mathrm{erg} \mathrm{s}^{-1}\right)$ to $22.2 \times 10^{-12} \mathrm{erg} \mathrm{s}^{-1} \mathrm{~cm}^{-2}\left(1.2 \times 10^{47} \mathrm{erg} \mathrm{s}^{-1}\right)$, with a constant photon index of 2.4 assuming a power-law slope.

J0946+1017 underwent a giant $\gamma$-ray flare on July 2nd, 2014, with a daily average $\gamma$-ray flux about 25 times greater than the average flux reported in the 2FGL catalogue (Ciprini 2014).

\section{DISCUSSION AND CONCLUSIONS}

J0946+1017 is the highest-redshift $\gamma$-ray emitting NLS1 known to date. It is one of the radio-loudest, and one of the few which show high-amplitude $\gamma$-ray flaring. Its Balmer decrement $\mathrm{H} \alpha / \mathrm{H} \beta \simeq 3$, implies little optical extinction. Its black hole mass, either derived from the Lorentzian fit $\left(M_{\mathrm{BH}}=1.6 \times 10^{8} M_{\odot}\right)$ or Gaussian fit $\left(M_{\mathrm{BH}}=1.9 \times 10^{8} M_{\odot}\right)$ based on the single-epoch scaling relation between Balmer line parameters and $\mathrm{BH}$ mass (Vestergaard \& Peterson 2006), is at the lower end of BH masses in typical blazars, but at the high end of the NLS1 distribution. This result agrees well with the estimates of $M_{\mathrm{BH}}$ based on $\mathrm{Mg}$ II lines (Shen et al. 2008; Rafiee \& Hall 2011; Shaw et al. 2012). Therefore J0946+1017 is an important cornerstone object bridging the two regimes. The monochromatic luminosity at $5100 \AA$ is estimated to be $\lambda L_{5100} \approx 1.2 \times 10^{45} \mathrm{erg} \mathrm{s}^{-1}$ from the $\mathrm{H} \beta$ luminosity based on the relation given in Zhou et al. (2006), as the observed flux at $5100 \AA$ 


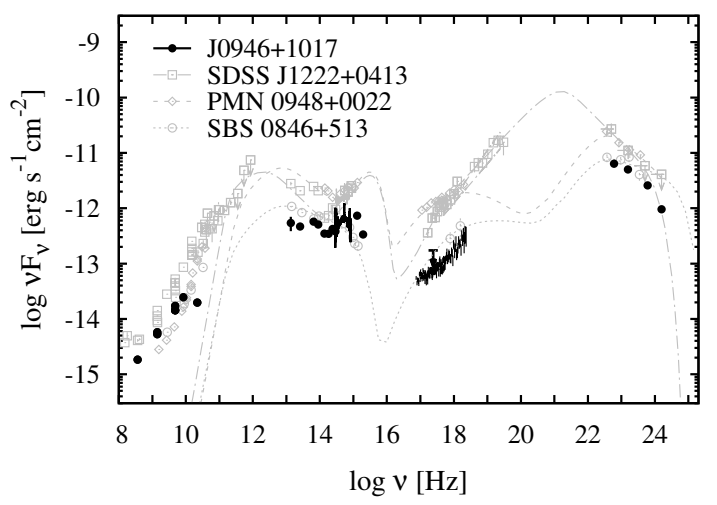

Figure 5. The SED of J0946+1017 (black) constructed with data from WISE, BOSS, RASS, XMM-Newton, the Fermi/LAT 4-year point source catalog (Acero et al. 2015), and data from the NASA/IPAC Infrared Science Archive and Extragalactic Database (NED). The data are nonsimultaneous and are corrected for Galactic reddening/absorption. The SED of three other $\gamma$-ray detected NLS1s, SDSS J1222+0413 (Yao et al. 2015), PMN J0948+0022 (Foschini et al. 2012) and SBS 0846+513 (D'Ammando et al. 2012; Sun et al. 2015) and their models are also plotted for comparison.

is likely contaminated by the beamed jet emission. With a bolometric correction of $k=9.8$ (McLure \& Dunlop 2004), the Eddington ratio of J0946+1017 then is $\sim 0.5$; typical of a NLS1.

While many of the radio-loud NLS1 galaxies have very compact radio emission in FIRST or in dedicated radio imaging (e.g. Komossa et al. 2006; Gu et al. 2015; Berton et al. 2018), J0946+1017 is remarkable in showing widely extended radio emission in FIRST, on a (projected) spatial scale of $120 \mathrm{kpc}$. Several other NLS1 galaxies with very extended radio emission have been identified (e.g. Gliozzi et al. 2010; Doi et al. 2012; Richards \& Lister 2015; Gabányi et al. 2018b; Rakshit et al. 2018; Singh \& Chand 2018), however, only few of them exceed 100 kpc (e.g., SDSS J103024.95+551622.7, Rakshit et al. 2018; SDSS J110006.07+442144.3, Gabányi et al. 2018b; and SDSS J094857.3+002225 with 395 kpc, Komossa et al. 2006).

The SED of J0946+1017 (Figure 5) broadly resembles that of other $\gamma$-ray NLS1s (with three peaks in the radio, ultraviolet and $\gamma$-ray bands, respectively, Yao et al. 2015), which have been well fitted by leptonic jet models, which require external Compton scattering in the high-energy regime, with seed photons from the accretion disk, broad-line region, and/or torus. The overall flatness of the X-ray spectrum implies the dominance of jet emission, but the XMM spectrum can be well fit by a two-component model including thermal emission at low energies. The IR variability on timescales of months to days implies that the emission is not from the dusty torus, but rather related to the jet. Multi-band monitoring of future high-amplitude flaring of J0946+1017 will provide us with important probes of the jet physics in the radio-loudest AGN.

\section{ACKNOWLEDGEMENTS}

We thank the anonymous referee for the very helpful comments and A. Lobanov for useful remarks. SY thanks A. Y. Yang for her help with plotting Fugire 2. SY acknowledges support by the KIAA-CAS Fellowship, which is jointly supported by Peking University and the Chinese Academy of Sciences. WJL acknowledges supports from the Natural Science Foundation of China (NSFC) grant No. 11703079 and the "Light of West China" Programme of Chinese Academy of Sciences (CAS). WY thanks the support from the National Science Foundation of China (NSFC-11703076) and the West Light Foundation of the Chinese Academy of Sciences (Y6XB016001). XBW thanks the supports by the Ministry of Science and Technology of China under grant 2016YFA0400703, the NSFC grants No.11721303 and 11533001 . This research made use of data taken from SDSS-BOSS, FIRST, WISE, CRTS, PanSTARRS, and XMM-Newton. This research has made use of data taken from TripleSpec on P200 telescope through the Telescope Access Program (TAP). This research has made use of the NASA/IPAC Infrared Science Archive and Extragalactic Database (NED), which are operated by the Jet Propulsion Laboratory, California Institute of Technology, under contract with the National Aeronautics and Space Administration.

\section{REFERENCES}

Abdo A. A., et al., 2009a, ApJ, 699, 976

Abdo A. A., et al., 2009b, ApJ, 707, L142

Abdo A. A., et al., 2010, ApJS, 188, 405

Acero F., et al., 2015, The Astrophysical Journal Supplement Series, 218, 23

Andrae R., et al., 2018, A\&A, 616, A8

Arnaud K. A., 1996, in Jacoby G. H., Barnes J., eds, Astronomical Society of the Pacific Conference Series Vol. 101, Astronomical Data Analysis Software and Systems V. p. 17

Becker R. H., White R. L., Edwards A. L., 1991, ApJS, 75, 1

Becker R. H., White R. L., Helfand D. J., 1995, ApJ, 450, 559

Berton M., et al., 2018, A\&A, 614, A87

Boroson T. A., Green R. F., 1992, ApJS, 80, 109

Chambers K. C., et al., 2016, arXiv e-prints,

Ciprini S., 2014, The Astronomer's Telegram, 6292

Cushing M. C., Vacca W. D., Rayner J. T., 2004, PASP, 116, 362

D'Ammando F., et al., 2012, MNRAS, 426, 317

D'Ammando F., Orienti M., Larsson J., Giroletti M., 2015, MNRAS, 452, 520

Dawson K. S., et al., 2013, AJ, 145, 10

Doi A., Nagira H., Kawakatu N., Kino M., Nagai H., Asada K., 2012, ApJ, 760,41

Douglas J. N., Bash F. N., Bozyan F. A., Torrence G. W., Wolfe C., 1996, AJ, 111, 1945

Drake A. J., et al., 2009, ApJ, 696, 870

Fey A. L., et al., 2015, AJ, 150, 58

Foschini L., 2011, in Narrow-Line Seyfert 1 Galaxies and their Place in the Universe. p. 24 (arXiv:1105.0772)

Foschini L., et al., 2012, A\&A, 548, A106

Gabanyi K., Moor A., Frey S., 2018a, in Revisiting Narrow-Line Seyfert 1 Galaxies and their Place in the Universe. p. 42 (arXiv:1807.05802)

Gabányi K. É., Frey S., Paragi Z., Järvelä E., Morokuma T., An T., Tanaka M., Tar I., 2018b, MNRAS, 473, 1554

Gliozzi M., Papadakis I. E., Grupe D., Brinkmann W. P., Raeth C., Kedziora-Chudczer L., 2010, ApJ, 717, 1243

Grupe D., Komossa S., Leighly K. M., Page K. L., 2010, ApJS, 187, 64

Gu M., Chen Y., Komossa S., Yuan W., Shen Z., Wajima K., Zhou H., Zensus J. A., 2015, ApJS, 221, 3

Healey S. E., Romani R. W., Taylor G. B., Sadler E. M., Ricci R., Murphy T., Ulvestad J. S., Winn J. N., 2007, ApJS, 171, 61

Helfand D. J., White R. L., Becker R. H., 2015, ApJ, 801, 26

Jiang N., et al., 2012, ApJ, 759, L31

Kalberla P. M. W., Burton W. B., Hartmann D., Arnal E. M., Bajaja E., Morras R., Pöppel W. G. L., 2005, A\&A, 440, 775

Komossa S., 2018, in Revisiting narrow-line Seyfert 1 galaxies and their place in the Universe. 9-13 April 2018. Padova Botan- 
ical Garden, Italy. Online at $<\mathrm{A}$ href="https://pos.sissa.it/cgibin/reader/conf.cgi?confid=328">https://pos.sissa.it/cgi-

bin/reader/conf.cgi?confid=328</A $>, \quad$ id. 15 . p. 15 (arXiv:1807.03666)

Komossa S., Voges W., Xu D., Mathur S., Adorf H.-M., Lemson G., Duschl W. J., Grupe D., 2006, AJ, 132, 531

Lähteenmäki A., Järvelä E., Ramakrishnan V., Tornikoski M., Tammi J., Vera R. J. C., Chamani W., 2018, A\&A, 614, L1

Laor A., Jannuzi B. T., Green R. F., Boroson T. A., 1997, ApJ, 489, 656

Linford J. D., Taylor G. B., Romani R. W., Helmboldt J. F., Readhead A. C. S., Reeves R., Richards J. L., 2012, ApJ, 744, 177

Mainzer A., et al., 2014, ApJ, 792, 30

Markwardt C. B., 2009, in Bohlender D. A., Durand D., Dowler P., eds, Astronomical Society of the Pacific Conference Series Vol. 411, Astronomical Data Analysis Software and Systems XVIII. p. 251 (arXiv:0902.2850)

McLure R. J., Dunlop J. S., 2004, MNRAS, 352, 1390

Osterbrock D. E., Pogge R. W., 1985, ApJ, 297, 166

Paliya V. S., Ajello M., Rakshit S., Mandal A. K., Stalin C. S., Kaur A., Hartmann D., 2018, ApJ, 853, L2

Petrov L., Hirota T., Honma M., Shibata K. M., Jike T., Kobayashi H., 2007, AJ, 133, 2487

Rafiee A., Hall P. B., 2011, ApJS, 194, 42

Rakshit S., Stalin C. S., Hota A., Konar C., 2018, ApJ, 869, 173

Rakshit S., Johnson A., Stalin C. S., Gandhi P., Hoenig S., 2019, MNRAS, 483, 2362

Richards J. L., Lister M. L., 2015, ApJ, 800, L8

Romano P., Vercellone S., Foschini L., Tavecchio F., Landoni M., Knödlseder J., 2018, MNRAS, 481, 5046

Schlafly E. F., Finkbeiner D. P., 2011, ApJ, 737, 103

Shaw M. S., et al., 2012, ApJ, 748, 49

Shen Y., Greene J. E., Strauss M. A., Richards G. T., Schneider D. P., 2008, ApJ, 680, 169

Singh V., Chand H., 2018, MNRAS, 480, 1796

Strüder L., et al., 2001, A\&A, 365, L18

Sun X.-N., Zhang J., Lin D.-B., Xue Z.-W., Liang E.-W., Zhang S.-N., 2015, ApJ, 798, 43

The Fermi-LAT collaboration 2019, arXiv e-prints, p. arXiv:1902.10045

Tsuzuki Y., Kawara K., Yoshii Y., Oyabu S., Tanabé T., Matsuoka Y., 2006, ApJ, 650, 57

Vanden Berk D. E., et al., 2001, AJ, 122, 549

Véron-Cetty M.-P., Véron P., Gonçalves A. C., 2001, A\&A, 372, 730

Véron-Cetty M.-P., Joly M., Véron P., 2004, A\&A, 417, 515

Vestergaard M., Peterson B. M., 2006, ApJ, 641, 689

Voges W., et al., 1999, A\&A, 349, 389

Wang J.-G., et al., 2009, ApJ, 707, 1334

White R. L., Becker R. H., 1992, ApJS, 79, 331

Wilson J. C., et al., 2004, in Moorwood A. F. M., Iye M., eds, Proc. SPIEVol. 5492, Ground-based Instrumentation for Astronomy. pp 1295-1305, doi:10.1117/12.550925

Wright E. L., et al., 2010, AJ, 140, 1868

Xu D., Komossa S., Zhou H., Lu H., Li C., Grupe D., Wang J., Yuan W., 2012, AJ, 143, 83

Yang H., et al., 2018, MNRAS, 477, 5127

Yao S., Yuan W., Zhou H., Komossa S., Zhang J., Qiao E., Liu B., 2015, MNRAS, 454, L16

Yuan W., Zhou H. Y., Komossa S., Dong X. B., Wang T. G., Lu H. L., Bai J. M., 2008, ApJ, 685, 801

Yuan W., Zhou H., Dou L., Dong X.-B., Fan X., Wang T.-G., 2014, ApJ, 782,55

Zhou H., Wang T., Yuan W., Lu H., Dong X., Wang J., Lu Y., 2006, ApJS, 166,128

Zuo W., Wu X.-B., Fan X., Green R., Wang R., Bian F., 2015, ApJ, 799, 189

van der Marel R. P., Franx M., 1993, ApJ, 407, 525

This paper has been typeset from a $\mathrm{T}_{\mathrm{E}} \mathrm{X} / \mathrm{L} \mathrm{A} \mathrm{E} \mathrm{X}$ file prepared by the author. 